\title{
Sociedad y escuela que deseamos: la dialéctica entre inclusión y exclusión social
}

\author{
Society and school we want: The dialectic between inclusion \\ and social exclusion
}

\author{
Leire Darretxe Urrutxi ${ }^{a}$ ORCID: 0000-0001-7468-7915 \\ Nekane Beloki ${ }^{\text {b }}$ ORCID: 0000-0002-2728-845X \\ Arantza Remiro ${ }^{c}$ ORCID: 0000-0001-8430-8405
}

Recibido: 11/12/2019 • Aprobado: 15/01/2020

Cómo citar: Darretxe Urrutxi, L., Beloki, B., \& Remiro, A. (2020). Sociedad y escuela que deseamos: la dialéctica entre inclusióny exclusión social. Ciencia y Educación, 4(1), 71-81. Doi: https://doi.org/10.22206/cyed.2020.v4i1.pp71-81

\section{Resumen}

La lucha por el derecho a la educación y por una educación inclusiva, que considera la diversidad como valor, cuenta con un desarrollo histórico que está avanzando con el paso del tiempo. Este artículo pretende aclarar dicha evolución mostrando la importancia de entender la relación existente entre la mirada que tiene la sociedad hacia la diversidad y la función de la educación y la escuela en el desafío de garantizar una educación inclusiva, equitativa y de calidad para todas las personas, sin ningún tipo de exclusión; por tanto, nos invita a reflexionar sobre el tipo de sociedad, educación y escuela que deseamos. En este proceso de avance hacia una sociedad y una escuela más justas resulta fundamental comprender la dialéctica entre inclusión y exclusión social, asumiendo un compromiso para avanzar hacia la sociedad y la escuela anheladas.

Palabras clave: educación inclusiva; educación equitativa; inclusión; sociedad; exclusión social.

\begin{abstract}
The struggle for the right to education and for an inclusive education that considers diversity as a value has a historical development that is advancing over time. This article aims to clarify this evolution by showing the importance of understanding the relationship between society's view of diversity and the role of education and school in the challenge of ensuring inclusive, equitable and quality education for all without exclusion. Therefore, it invites us to reflect on the type of society, education and school we want. In this process it is essential to understand the dialectic between inclusion and social exclusion, assuming a commitment to advance towards the desired society and school.
\end{abstract}

Keywords: Inclusive education; equitable education; inclusion; society; social exclusion.

\footnotetext{
a Universidad del País Vasco/Euskal Herriko Unibertsitatea (UPV/EHU), España. Correo-e: leire.darretxe@ehu.eus

b Universidad del País Vasco/Euskal Herriko Unibertsitatea (UPV/EHU), España. Correo-e: nekane.beloki@ehu.eus

${ }^{c}$ Observatorio del Tercer Sector Social de Euskadi, España. Correo-e: arantzanestor@bizkaia.eu
} 


\section{Introducción}

En muchas ocasiones hemos escuchado la interrogante “'yo seré normal?”. Por una parte, la perspectiva de la homogeneidad distingue entre alumnado normal y alumnado categorizado como diferente (necesidades educativas especiales, inmigrante, etc.); sin embargo, la lógica de la heterogeneidad reconoce y acepta la diversidad de todas las personas (Muntaner, Roselló \& De la Iglesia, 2016). Siguiendo esta segunda postura se entiende que cada persona es diferente a las demás y esa diversidad es un elemento enriquecedor y un valor positivo para la sociedad.

Así, la diversidad existente en la sociedad es una realidad innegable que se está manifestando cada vez de manera más notable en los centros educativos. La Organización de las Naciones Unidas para la Educación, la Ciencia y la Cultura (UNESCO, 2017b, p. 7) define la diversidad como "diferencias entre las personas, que pueden ser en función de la raza, etnia, género, orientación sexual, idioma, cultura, religión, capacidad mental y física, clase y situación migratoria”. Sin pretender caer en categorizaciones, lo que resulta irrenunciable es que cada persona es única e irrepetible.

La diferencia es la norma en el mundo en el que vivimos, en las especies y en la sociedad. La escuela forma parte de la sociedad y en ella hay personas diversas que comparten distintas formas de aprender y de enseñar (Pérez, 2017, p. 17).

No obstante, cabe destacar que la diversidad no siempre ha sido entendida como valor ni como oportunidad de aprendizaje, condicionando a numerosas personas a vivir prácticas de escolaridad excluyentes.

Es posible ofertar un modelo segregado o diferenciado de educación, en el que los alumnos se escolarizan de acuerdo con determinadas características personales, de muy distinto tipo. Aparecen escuelas para niños, por un lado, y para niñas, por otro, para alumnos con necesidades educativas especiales, para alumnos con alta capacidad intelectual, para inmigrantes (por nacionalidades o lenguas), para indígenas, para los de determinada religión, para los pertenecientes a diferente clase social... En fin, la gama es variada según el país y la ideología del gobierno que lo dirige (Casanova, 2017, p. 81).

A lo largo de la historia son conocidas algunas críticas hacia el sistema educativo, por ejemplo la realizada por Tonucci (2005) en la década de los ańos setenta sobre el sistema educativo conservador denominado "la máquina de la escuela", en donde se dejaba patente que hacia la carrera del bienestar, la cultura, la dignidad y el poder únicamente se dirigían los estudiantes que salían de la escuela considerados "normales" o, en su caso, "homogéneos", mientras que una multitud de personas se perdían en el camino siendo considerados como desechos.

Esto quiere decir que la mirada que se tiene de la diversidad y la función que tiene la educación y la escuela como institución social suponen un pilar fundamental en la vida de las personas, estando muy relacionado con las circunstancias histórico-sociales en las que se producen. Así, "la educación puede ser un factor de cohesión si procura tener en cuenta la diversidad de los individuos y de los grupos humanos y al mismo tiempo evita ser a su vez un factor de exclusión social" (Delors, 1996, p. 59).

¿Qué tipo de sociedad, educación y escuela queremos?

Con respecto a la sociedad, Lodi (1977) formulaba una analogía respecto a la condición de la infancia en la escuela autoritaria y la de la clase obrera en la fábrica, argumentando que ambos son agentes pasivos sin participación:

La condición de un niño en la escuela no difiere mucho de la de un obrero en la fábrica... El obrero trabaja en la cadena de montaje sin participación... Dentro del aula que, como decía, recuerda una cárcel o una fábrica, sólo por su aspecto, el esquema en el que se desenvuelve el alumno es simple, funcional, rígido y terrible: explicación, repetición, nota; dictado, redacción, problema y nota. Todo, dentro y fuera del colegio, está predispuesto a anular al alumno en cuanto ser capaz de pensar... (Lodi, 1977, p. 15).

También, a finales de la década de los ochenta, Bauman (2004) acuñó el término modernidad líquida para reflejar que vivimos en una sociedad líquida y 
maleable y, además, dejaba patente que somos seres reflexivos comprometidos con la política de la vida. A esta lectura teórica de la sociedad hay que sumar la idea de que nos encontramos en la sociedad de la información o del conocimiento o en la sociedad red:

El concepto de sociedad de la información o del conocimiento es sencillamente una extrapolación tecnológica de la sociedad industrial, habitualmente identificada con la cultura occidental de modernización. El concepto de la sociedad red cambia el énfasis a la transformación organizativa y a la aparición de una estructura social globalmente interdependiente, con sus procesos de dominación y contra-dominación. También nos ayuda a definir los términos del dilema fundamental de nuestro mundo: el dominio de los programas de una red global de poder o, en su lugar, la emergencia de una red de cultura interactivas, unificadas por la creencia común en el valor de uso de compartir (Castells, 2006, p. 73).

Siguiendo a Castells (2018), vivimos una crisis de la democracia liberal en la que están aumentando las desigualdades sociales, provocando la polarización; es decir, "los ricos son cada vez más ricos, sobre todo en la cúspide de la pirámide, y los pobres cada vez más pobres" (Castells, 2018, p. 20). Así que nos anima a vivir en un caos creativo en el que vayamos reconstruyendo de abajo a arriba el tejido de nuestras vidas tanto en lo personal como en lo social. También Nussbaum (2018, p. 227) señala que "el mundo contiene desigualdades que son moralmente alarmantes, y la brecha entre los países más ricos y los más pobres es cada vez mayor".

En las sociedades y en las Ciencias Sociales se está dando un giro dialógico como clave para lograr mayores niveles de democracia e igualdad social (Aubert et al., 2008). Además, como afirma Casanova (2018), nos encontramos ante una vida de incertidumbre caracterizada por el cambio acelerado de situaciones sociales y vitales. En este sentido, resulta muy interesante la reflexión que plantean Aubert et al. (2008) en la que diferencian tres concepciones distintas y, en gran medida, contrapuestas, del funcionamiento de la sociedad: sociedad industrial con una concepción objetivista; sociedad industrial con una concepción constructivista; y la sociedad de la información o del conocimiento con una concepción comunicativa.

Estamos pues ante un nuevo modelo social, un tipo de organización social que tiene unas características determinadas que tenemos que considerar cuando hablamos y reflexionamos sobre lo que es la educación. Como señala McLaren (2005, p. 80) "vivimos en un curso peligroso de la historia en el que la democracia se encuentra en retirada", siendo el papel de los educadores y educadoras fundamental para alentar a los estudiantes a cuestionar las injusticias sociales existentes.

Ante este panorama, Apple (2013) se cuestiona si la educación puede cambiar a la sociedad, argumentando que "los educadores críticos han sido guiados por una preocupación permanente relacionada con el rol de la educación no sólo porque reproduce la noción de dominación, sino también en su rol para desafiar a dicha dominación" (p. 31). Así, Apple \& Beane (2005) comparten la idea de que el ideal democrático de educación debe influir en las escuelas públicas. "No dudes de que un pequeño grupo de ciudadanos reflexivos comprometidos puede cambiar el mundo; de hecho, es la única cosa que siempre lo ha cambiado" (Mead, citado en Peterson, 2005, p. 123). Y como señalan Calderón \& Verde (2018, p. 68) "el mundo es así hasta que decidamos que cambie, y cada día puede constituir una fiesta para que esas transformaciones necesarias se produzcan”.

En palabras de Shirley, en el prólogo del libro de McLaren \& Kincheloe (2008, p. 14), Pedagogía Crítica: de qué hablamos, dónde estamos: "estamos en las escuelas, estamos en las aulas, estamos en los programas de formación del profesorado, estamos en las organizaciones de base, estamos en la sociedad". Por consiguiente, cualquier persona que cree en la democracia debe considerar el papel político de la educación, ya que moldea la manera de pensar, sentir y sońar de las personas (Giroux, 2015). Según Echeita (2017), se trata de una cuestión de carácter político sobre el proyecto de sociedad en el que queremos vivir. Esto es, la educación es un acto revolucionario, es decir, supone una batalla política (Calderón \& Verde, 2018). Por ejemplo, Giroux (2009) afirma que la educación es 
una práctica moral y política, y reclama que los educadores y educadoras necesitamos una "Pedagogía de la Ruptura": Y según Giroux (2016, p. 19) "una pedagogía cosmopolita, imaginativa, afirmativa de lo público que reclame una interacción crítica y comprometida con el mundo en que vivimos, mediada por la responsabilidad para desafiar estructuras de dominación y para aliviar el sufrimiento humano”.

Ante este panorama, la educación inclusiva nos invita a reflexionar sobre el mundo en el que vivimos, el que preferimos, y nuestro papel en la configuración de ambos (Slee, 2012). Así, Carbonell (2017, p. 142) se cuestiona “¿qué se debe aprender en la escuela y qué modelo de ciudadano y ciudadana se quiere formar?”. Si apostamos por una sociedad democrática, todas las personas tienen que ser aceptadas respetando las diferencias personales como un elemento enriquecedor (Casanova, 2017). En esta línea, Parrilla (2013) matiza que la sociedad ha de ser cada vez más equitativa, cohesionada e inclusiva, de ahí que sea fundamental plantearse el papel que tiene la educación para favorecer la construcción de dicha sociedad. En palabras de Rubia (2017), el debate debería abordar lo que deseamos:

Un sistema inclusivo y equitativo que contribuya a la cohesión de la sociedad o un sistema excluyente e injusto que favorece una sociedad más fragmentada, y que distribuye sus recursos y oportunidades de forma desigual, al estilo del llamado efecto Mateo (p. 91).

En definitiva, todo esto nos lleva a reconocer las desigualdades existentes en una sociedad que está en constante cambio y que, por lo tanto, la escuela también debe enfrentarse a las desigualdades que existen en nuestros contextos, siendo la educación una de las armas más potentes con las que contamos.

\section{Desarrollo histórico desde la exclusión a la educación inclusiva}

Desde la exclusión a la inclusión, pasando por la integración, el camino a avanzar en la búsqueda de una escuela y, por lo tanto, de una sociedad más justa y equitativa está siendo largo y con obstáculos que solventar (Fiuza \& Sierra, 2017).
Históricamente han existido diferentes etapas de desarrollo, que pueden variar en cada país, con respecto a la manera en la que los sistemas educativos atienden a la infancia con dificultades, tratándose de un acercamiento gradual, desde la exclusión hacia la educación especial, la integración y la educación inclusiva (Ainscow, 2012). Por ejemplo, en cuanto al alumnado con discapacidad se han ido dando diferentes modelos educativos como respuesta a dichas personas (Etxabe, López-Vélez \& Soria, 2018; García Rubio, 2017; UNICEF, 2017):

- Exclusión: al alumnado se le niega el acceso a la educación en cualquier forma, considerando que no tienen ningún derecho a recibir educación.

- Segregación o separación: se sustenta en el modelo de los déficits de la persona. La educación a este alumnado se le proporciona en entornos separados diseńados específicamente y estando aislados del alumnado sin discapacidad. Así se consiguen grupos homogéneos ante los cuales se piensa que es más sencillo enseñar.

- Integración: el alumnado con discapacidad se tiene que adaptar al sistema existente en la escuela y a un modelo de ser y hacer "normalizado". Las necesidades son del alumnado y no de la escuela ni del contexto.

- Inclusión: entornos educativos que adaptan las estructuras, los métodos de enseñanza y el curriculum, así como la cultura, la política y la práctica de dichos entornos para que sean accesibles para todo el alumnado, sin discriminación. El alumnado que presente algún tipo de discapacidad tiene derecho a estar en las aulas ordinarias y la diversidad es considerada como valor educativo, apostándose por la heterogeneidad.

Arnaiz (2012) sugiere que se debe indagar lo que sucede en las escuelas, es decir, recomienda que se debe analizar si las dificultades de aprendizaje que experimenta el alumnado tienen relación con procesos implícitos en las prácticas escolares que se llevan a cabo en el centro. Así, conviene subrayar que existen dos modelos confrontados de carácter teórico a la hora de conceptualizar las necesidades del alumnado con discapacidad (Arnaiz, 2011; 2012; UNICEF, 2014): 
La visión individual o modelo del déficit: entiende que el problema es el niño o la niña con discapacidad, lo que lleva a que esa persona tenga una etiqueta de "especial".

La visión o modelo curricular: entiende que el problema está en el sistema en vez de en el niño o la niña con discapacidad. Cualquier niño o niña puede experimentar dificultades en la escuela, no solo los que tienen discapacidades. Estas dificultades pueden indicar en qué forma se puede mejorar la enseńanza y estas mejoras pueden favorecer a todos los demás. El profesorado debe recibir apoyo para convertir su práctica en más inclusiva, ya que se entiende que el alumnado aprende de manera diferente y los centros deben responder a las necesidades de todo el alumnado.

En síntesis, se va implantando un modelo que entiende el origen interactivo de los problemas de aprendizaje al desviar el foco de atención únicamente centrado en el alumnado hacia los procesos de enseñanza y aprendizaje, pasando así de un modelo individual a un modelo curricular (Arnaiz, 2011) o también llamado "paradigma organizativo" (Arnaiz, De Haro \& Azorín, 2018). Por consiguiente, evolucionar hacia sistemas educativos más inclusivos exige un cambio en las maneras de pensar, superando el modelo del déficit y posicionándose en la importancia de analizar las barreras al aprendizaje y a la participación de los sistemas escolares (Booth \& Ainscow, 2011 citado por Arnaiz, De Haro \& Azorín, 2018).

Y este cambio no solo alude a las personas con discapacidad. A lo largo de la historia diferentes aspectos han causado discriminación o exclusión: el color de la piel, el sexo, la lengua de origen, la religión, el origen nacional, el origen social, la situación económica, la pertenencia a una minoría étnica (Rubia, 2017). Así, en 1960, una niña llamada Ruby Bridges Hal se convirtió en la primera afroamericana que asistió a una escuela hasta entonces únicamente para personas de raza blanca, siendo su maestra una de las herramientas fundamentales para que ella dejara de ser marginada (Calderón \& Verde, 2018). Este ejemplo se puede extender a otros muchos, ya que como señala Parrilla (2002) existe un camino compartido en la respuesta escolar que se ha ido desarrollando en los últimos años en diferentes fases, considerando la clase social, el grupo cultural, el género y la discapacidad.

Tabla 1. De la exclusión a la inclusión: un camino compartido

\begin{tabular}{|c|c|c|c|c|}
\hline & Clase Social & Gr. Cultural & Género & Discapacidad \\
\hline 1.Exclusión & No escolarización & No escolarización & $\begin{array}{c}\text { No } \\
\text { escolarización }\end{array}$ & $\begin{array}{c}\text { Infanticidio/ } \\
\text { Internamiento }\end{array}$ \\
\hline 2.Segregación & Escuela Graduada & Esc. Puente & $\begin{array}{c}\text { Esc. Separadas: } \\
\text { Niñas }\end{array}$ & Esc. Especiales \\
\hline 3.Integración & $\begin{array}{c}\text { Comprehensividad } \\
(50-60)\end{array}$ & $\begin{array}{c}\text { E. Compensatoria } \\
\text { E. Multicultural } \\
(80)\end{array}$ & $\begin{array}{c}\text { Coeducación } \\
\text { (70) }\end{array}$ & $\begin{array}{c}\text { Integración } \\
\text { E (60) }\end{array}$ \\
& Educ. Inclusiva & $\begin{array}{c}\text { Educ. Inclusiva } \\
\text { (Ed. intercultural) }\end{array}$ & Educ. Inclusiva & $\begin{array}{c}\text { Educ. } \\
\text { Inclusiva }\end{array}$ \\
\hline 4.Reestructuración & & & \\
\hline
\end{tabular}

Fuente: Parrilla (2002, p. 15).

Actualmente, también existen muchos grupos sociales que están amenazados por la exclusión o expuestos a vivir formas de vida precarias (Castel, 2014). Por consiguiente, la reducción de las desigualdades y la exclusión social siguen siendo desafíos cruciales para el futuro. Muestra de esto es el discurso de Malala Yousafzai, Premio Nobel de la Paz, ante la ONU, el 12 de julio de 2013, que dio la vuelta al mundo. Esta adolescente paquistaní de 16 años, a quien intentaron matar en el año 2012 con una bala, desafió al régimen talibán reclamando el derecho de las niñas a la educación.

La experiencia que explica Malala sigue siendo una realidad que impide que actualmente millones de niños y niñas ejerzan el derecho a la educación (Azorín, 2018). A su vez, un Informe del Comité sobre los derechos de las personas con discapacidad de la ONU, tras una investigación llevada a cabo a partir de una denuncia, condena a España por vulnerar el artículo 24 del tratado internacional que garantiza el derecho a la educación inclusiva y de calidad, argumentando que se ha perpetuado un patrón estructural de segregación y exclusión basado en la discapacidad a través de un modelo médico. 
El Comité considera que la información disponible revela violaciones al derecho a la educación inclusiva y de calidad principalmente vinculadas a la perpetuación, pese a las reformas desarrolladas, de las características de un sistema educativo que continua excluyendo de la educación general, particularmente a personas con discapacidad intelectual o psicosocial y discapacidades múltiples, con base en una evaluación anclada en un modelo médico de la discapacidad y que resulta en la segregación educativa y en la denegación de los ajustes razonables necesarios para la inclusión sin discriminación en el sistema educativo general (Comité sobre los derechos de las personas con discapacidad, 2017, p. 7).

El informe señala que en España existe un sistema de educación paralelo que consiste en los centros de educación especial o en las aulas especiales dentro de los centros ordinarios, perpetuando un sistema discriminatorio de segregación de las personas con discapacidad. Por tanto, el comité recomienda abolir el sistema separado de educación para estudiantes con discapacidad, tanto en los centros especiales como en una unidad dentro de la misma escuela.

Según Calderón (2018), este informe ha tenido un impacto considerable y están haciéndose públicos más testimonios de vulneración del derecho a la educación inclusiva. En concreto, Calderón \& Verde (2018) apuestan por terminar con el apartheid educativo que existe en muchas realidades y que tiene como consecuencia que muchas personas hayan sido deshumanizadas a lo largo de la historia. Según Arnaiz (2012, p. 28) "vivimos en un mundo marcado por la exclusión, la desigualdad y la marginación, en una sociedad tantas veces injusta, donde los derechos humanos son vulnerados".

Ante esta realidad, las personas que viven en sus propias carnes estos procesos de marginación suelen ser las primeras en reclamar políticas y prácticas inclusivas (Echeita, 2017) y, en muchos casos, también son las propias familias las que luchan por los derechos de sus hijos e hijas, por ejemplo, por el derecho de habitar en escuelas ordinarias (Calderón \& Verde, 2018).

\section{La dialéctica entre inclusión y exclusión social}

No podemos reflexionar sobre la inclusión sin referirnos a la exclusión social y según Castel (2014) vivimos en una sociedad en la que existe una dinámica de precarización que generaliza los riesgos de exclusión. De este modo, nos encontramos en un contexto de incertidumbre sin poder saber qué sucederá mañana.

Es necesario analizar estas situaciones en términos de proceso o de recorrido y no conformarse con emplear el término de exclusión. Por ejemplo, hay personas que trabajan, tienen un jefe y un sueldo, pero bajo condiciones tan precarias y tan degradadas que en seis meses pueden verse totalmente desprovistos de su nivel de vida. Incluso alguien que ocupe una vivienda cuyo alquiler consigue pagar a duras penas, puede verse desprovisto de vivienda al tener un contrato laboral de seis meses de duración. Es a esta nebulosa de situaciones inestables y frágiles, a la que hay que referirse para posicionarse con respecto a la exclusión de la sociedad actual (Castel, 2014, p. 18).

Como señala Karsz (2004), la exclusión incita a reflexionar sobre las condiciones de coexistencia que se crean entre grupos, categorías y clases sociales con posturas e intereses tanto personales como colectivos contrarios.

La categoría de exclusión insiste para que nos ocupemos de los excluidos, para que estos cesen de serlo a fin de que la sociedad real coincida finalmente con la sociedad ideal, con tal de que los humanos reales se correspondan con los humanos ideales (Karsz, 2004, p. 209).

Castel (2004) explica tres formas de exclusión: a) la primera supone una sustracción completa de la comunidad: la deportación, el destierro, la matanza o el genocidio, que es la última forma de erradicación total; b) una segunda forma consistente en crear espacios cerrados en el seno de la comunidad, pero separadas de esta: los manicomios, las prisiones, los guetos, las leproserías, etc.; c) una tercera forma que supone dotar a ciertas poblaciones de un estatuto especial permitiéndoles coexistir en la comunidad, pero privándoles de derechos y de participación en determinadas 
actividades sociales. Según este mismo autor, en la actualidad no cree que debamos temer que ocurra la primera modalidad de exclusión, que es la más radical. La segunda forma de exclusión (el relegamiento a espacios particulares) parece menos improbable; sin embargo, la tercera forma de exclusión (atribuir un estatuto especial a ciertas categorías de la población) supone la principal amenaza, sobre todo, a través de las políticas de discriminación positiva.

Desde el momento en que se comienza a asignar un estigma a través del encierro, no en un espacio vallado, sino en una etiqueta que discrimina negativamente a las personas a las que se aplica cuando, en realidad, dicha etiqueta quería discriminarlas positivamente. Yo planteo este interrogante, que merece una discusión abierta: no creo que se pueda prescindir de políticas de discriminación positiva, pero ¿qué hacer para evitar que se transformen en lo contrario? (Castel, 2004, p. 69).

Además de todo lo comentado, debemos considerar que la exclusión social tiene un carácter pluridimensional y multifactorial (Garrido, 2012); procesual, dinámico (Camacho, 2014) y estructural (Sánchez \& Jiménez, 2013). Por lo tanto, la exclusión social supone una realidad compleja puesto que existen dimensiones interrelacionadas entre sí; carencias en multitud de ámbitos; falta de acceso a recursos y necesidades básicas; y, falta de participación en la dinámica social (Camacho, 2014). Y como indica Castel (2004, p. 67) "las exclusiones son formas de discriminación negativa que obedecen a reglas estrictas de construcción, en una sociedad dada”.

Asimismo, Sen (2000) examina críticamente la idea de exclusión en el contexto de pobreza como privación de capacidades básicas, siendo relevante analizar el vínculo entre exclusiones en diferentes ámbitos de las interacciones interindividuales e interfamiliares. En otras palabras, la inclusión y la exclusión son fenómenos que operan recíprocamente y han de abordarse de forma dialéctica (Echeita et al., 2014; Escudero \& Martínez, 2011; Garrido, 2012). Además, esta relación dialéctica está en permanente tensión:
Por ejemplo, no pocas veces se avanza en la inclusión educativa de algunos alumnos y se retrocede en la de otros, o se reducen algunas barreras que limitan la presencia o la participación de ciertos alumnos (por ejemplo, con políticas de escolarización más inclusivas), pero se refuerzan otras que les perjudican (por ejemplo, a través de modos de evaluar más uniformes para mejorar el rendimiento), lo que contribuye a configurar otro de los aspectos definitorios de la inclusión, cuál es su carácter dilemático (Echeita, 2013, p. 108).

De ahí que los conceptos de inclusión y exclusión sean conceptos útiles para repensar políticas y prácticas en diferentes ámbitos de la vida, como el educativo, social, económico, laboral, urbanístico, etc. (Echeita et al., 2014). De la misma manera, para avanzar hacia sociedades más inclusivas y justas resulta necesaria una perfecta coordinación con las reformas establecidas en diferentes ámbitos de la política pública, así como el diálogo y la colaboración con todas las partes interesadas (UNESCO, 2012). En esta línea, la educación inclusiva debe construir análisis de la exclusión para poder cuestionar relaciones culturales y sociales basadas en la opresión y poder promover la inclusión (Slee, 2012).

La educación inclusiva, que rechaza la lástima y la caridad, nos hace a todos más cultos socialmente y nos enseña que la injusticia no es una característica de las leyes de la naturaleza. La injusticia y la exclusión se construyen y sostienen por las elecciones que hacen personas con poder. La educación inclusiva no es, en consecuencia, una formulación de ideas acerca de las necesidades educativas especiales, sino una teoría y una táctica de la reforma educativa y social (Slee, 2012, pp. 163 y164).

Los datos actuales resultan alarmantes, ya que según la UNESCO (2017a) en el año 2015 había 264 millones de niños, niñas y jóvenes que no iban a la escuela, es decir, sin escolaridad en edad de cursar la enseñanza primaria o secundaria. Debemos ser conscientes de que esto supone un fracaso, ya que la educación es una responsabilidad compartida. 
Según otro informe de la UNESCO (2018), hay 1.8 billones de jóvenes entre la edad de 10 y 24 años en el mundo, lo que supone una oportunidad grande para el cambio positivo; pero, uno de cada 10 vive en zonas de conflicto y 24 millones están fuera de la escuela. Otras cuestiones que afectan negativamente son la inestabilidad política, la falta de empleo y el espacio limitado para la participación política y civil.

La transformación de la sociedad basándose en la justicia social resulta un desafío, por lo que los sistemas educativos actuales deben enfrentarse a la lucha contra la exclusión (Arnaiz, 2012). De ahí que uno de los retos para la educación de todos los países radica en ofrecer una educación inclusiva y de calidad (Rodríguez, Jiménez \& Sánchez, 2018). Así, la inclusión y la equidad son los principios básicos que deben orientar las políticas, los planes y las prácticas educativas para poder trabajar por una sociedad más justa (UNESCO, 2017b).

Una sociedad democrática es la que acepta a todo tipo de personas, valora sus aportaciones en cualquiera de los campos de actuación posible y desde cualquier enfoque ideológico, respeta las diferencias personales como un elemento que enriquece al resto de los grupos, protege las culturas minoritarias como expresión razonable de esas diferencias, permite y favorece la convivencia entre individuos y grupos distintos; en definitiva, un todo social que se complementa mediante la participación y las aportaciones de todos y cada uno de sus miembros y resulta beneficioso para ellos (Casanova, 2018, p. 43).

En definitiva, si hablamos de educación inclusiva es porque millones de niños y niñas a nivel mundial están excluidos y excluidas (Echeita et al., 2014), es decir, todavía existe una educación escolar que excluye al alumnado a lo largo de su proceso educativo (Echeita, 2017). Por esto, la educación inclusiva se justifica como una lucha contra la exclusión y el fracaso, adoptando un compromiso político por un mundo más justo (Azorín, 2017). Desde este planteamiento teórico, finalmente, no podemos concebir una educación de calidad, integral y justa para y con las personas, sin que se matice el adjetivo de "inclusiva” (Echeita et al., 2014).
Tabla 2. Relevancia del adjetivo "inclusiva" en la educación

\begin{tabular}{|c|c|}
\hline $\begin{array}{l}\text { Arnaiz \& Azorín } \\
\text { (2014, p. 229) }\end{array}$ & $\begin{array}{l}\text { "Ha de advertirse que no hay calidad sin } \\
\text { inclusión, pues en una sociedad democráti- } \\
\text { ca la educación o es inclusiva o no se puede } \\
\text { llamar educación" }\end{array}$ \\
\hline $\begin{array}{c}\text { Casanova } \\
(2018, \text { p. } 49)\end{array}$ & $\begin{array}{l}\text { "La educación en una sociedad democráti- } \\
\text { ca, o es inclusiva, o no es educación" }\end{array}$ \\
\hline $\begin{array}{l}\text { Bristol City Council, } \\
\text { citado en Escudero \& } \\
\text { Martínez } \\
\text { (2011, p. } 88)\end{array}$ & $\begin{array}{l}\text { "La educación inclusiva es la única edu- } \\
\text { cación moralmente defendible" }\end{array}$ \\
\hline
\end{tabular}

Fuente: elaboración propia.

\section{Conclusiones}

Si bien la diversidad del ser humano es una realidad innegable, se debe matizar que la sociedad ha utilizado y continúa utilizando diversos argumentos para llevar a cabo prácticas excluyentes hacia ciertas personas, por ejemplo, personas con discapacidad y otros colectivos en situación de vulnerabilidad. Preguntarse sobre qué tipo de sociedad, educación y escuela deseamos para nuestros hijos, hijas, hermanos, hermanas y un largo etcétera supone un proyecto político y ético ante el que debemos responder.

Desde la exclusión, segregación, integración hacia la inclusión se está produciendo una evolución sobre diversas formas de responder a la diversidad que varían según el contexto político, cultural, económico, etc., de cada sociedad. Actualmente se asume que una educación inclusiva supone entender que la escuela debe adaptarse a las necesidades de todo el alumnado, alejándose de una visión individual y exigiendo cambios en el sistema educativo. Este desafío de garantizar el derecho a una educación inclusiva nos lleva a plantearnos, entre otros aspectos, la dinámica entre exclusión e inclusión en cada sociedad concreta para asumir un compromiso con la lucha contra la exclusión, siendo capaces de ir avanzando hacia una sociedad y una escuela donde podamos convivir y aprender de la diversidad. 
Ante este dilema nos situamos en la perspectiva de la Teoría crítica de la educación y, en concreto, como señala Carbonell (2017), en las pedagogías críticas, entendiendo que el abanico tanto epistemológico como conceptual es muy amplio, ya que se incluyen todos los relatos con una visión crítica de la sociedad actual o un análisis crítico de la sociedad que abogan por el cambio. Así, por ejemplo, algunos referentes del ámbito de la sociología, como Robert Castel, Manuel Castells, Zygmunt Bauman, Jünger Habermas; y del ámbito de la educación, como Paulo Freire, Henry Giroux, Shirley Steinberg, Peter McLaren, Michael Apple, Joe Kincheloe ofrecen propuestas para continuar luchando por la transformación de la sociedad.

Desde las aportaciones de estas personas podemos plantear nuestra apuesta ideológica asumiendo sus presupuestos de compromiso político, erradicación del enriquecimiento continuo solo de las personas más ricas, la importancia de construir las sociedades de abajo arriba y la necesidad de desafiar el rol de denominación que algunos planteamientos otorgan a la educación. La opción por estos planteamientos tiene, para nosotras, un origen en la justicia social, la cual se plasma desde un marco de derechos y no de caridad.

\section{Referencias}

Ainscow, M. (2012). Haciendo que las escuelas sean más inclusivas: lecciones a partir del análisis de la investigación internacional. Revista De Educación Inclusiva, 5(1), 39-49.

Apple, M.W. \& Beane, J.A. (2005). Escuelas democráticas. Madrid: Morata.

Apple, M.W. (2013). Creando educación democrática en tiempos neoliberales y neoconservadores. Praxis Educativa, $17(1$ y 2), 27-35.

Arnaiz, P. (2011). Luchando contra la exclusión: buenas prácticas y éxito escolar. Innovación Educativa, (21), 23-35.

Arnaiz, P. (2012). Escuelas eficaces e inclusivas: cómo favorecer su desarrollo. Educatio Siglo XXI: Revista De La Facultad De Educación, 30(1), 25-44.

Arnaiz, P. \& Azorín, C. (2014). Autoevaluación docente para la mejora de los procesos educativos en escuelas que caminan hacia la inclusión. Revista Colombiana De Educación, 67, 227-245.
Arnaiz, P. , De Haro, R. \& Azorín, C. (2018). Redes de apoyo y colaboración para la mejora de la educación inclusiva. Profesorado: Revista De Curriculum Y Formación Del Profesorado, 22(2), 7-27.

Aubert, A. et al. (2008). Aprendizaje dialógico en la Sociedad de la Información. Barcelona: Hipatia.

Azorín, C. (2017). Análisis de instrumentos sobre educación inclusiva y atención a la diversidad. Revista Complutense De Educación, 28(4), 1043-1060.

Azorín, C. (2018). Abriendo fronteras para la inclusión: la ecología de la equidad. Revista de Educación Inclusiva, 11(1), 213-228.

Bauman, Z. (2004). Modernidad Líquida. Buenos Aires: Fondo de Cultura Económica.

Bristol City Council. (2003). Bristol Inclusion Standard. Good practice guidance for schools. Raising Achievement and Promoting Equality. https:// www.uam.es/personal_pdi/stmaria/sarrio/ DOCUMENTOS, \%20ARTICULOS, \%20 PONENECIAS,/Index_para_la_inclusion_-_ Bristol_City.pdf

Calderón, I. (2018). Deprived of human rights. Disability \& Society, 33(10), 1666-1671.

Calderón, I. \& Verde, P. (2018). Reconocer la diversidad: textos breves e imágenes para transformar miradas. Barcelona: Octaedro.

Camacho, J. (2014). Exclusión social. Eunomía. Revista en Cultura de la Legalidad, 7, 208-214.

Carbonell, J. (2017). Pedagogías del siglo XXI: alternativas para la innovación educativa. Barcelona: Octaedro.

Casanova, M.A. (2017). Educación inclusiva en las aulas. Madrid: La Muralla.

Casanova, M.A. (2018). Educación inclusiva: ¿Por qué y para qué? Revista Portuguesa De Educação, 31(Especial), 42-54.

Castel, R. (2004). Encuadre de la exclusión. En S. Karsz, La exclusion: bordeando sus fronteras. Definiciones y matices (pp. 55-86). Barcelona: Gedisa.

Castel, R. (2014). Los riesgos de exclusión social en un contexto de incertidumbre. Revista Internacional De Sociología, 72(Extra_1), 15-24. 
Castells, M. (2006). Informacionalismo, redes y sociedad red: una propuesta teórica. En M. Castells (Ed.), La Sociedad Red: una visión global (pp. 27-75). Madrid: Alianza.

Castells, M. (2018). Ruptura: la crisis de la democracia liberal. Madrid: Alianza.

Comité sobre los derechos de las personas con discapacidad (2017). Informe de la investigación relacionada con Espańa bajo el artículo 6 del Protocolo Facultativo. Disponible en: https:// tbinternet.ohchr.org/_layouts/treatybodyexternal/Download.aspx?symbolno=CRPD\% $2 \mathrm{fC} \% 2 \mathrm{f} 20 \% 2 \mathrm{f} 3 \&$ Lang=en

Delors, J. (1996). La educación encierra un tesoro. Madrid: Santillana.

Echeita, G. (2013). Inclusión y exclusión educativa: de nuevo, "voz y quebranto". REICE: Revista Electrónica Iberoamericana Sobre Calidad, Eficacia $Y$ Cambio En Educación, 11(2), 99-118.

Echeita, G. (2017). Educación inclusiva. sonrisas y lágrimas. Aula Abierta, 46, 17-24.

Echeita, G. et al. (2014). Reflexionando en voz alta sobre el sentido y algunos saberes proporcionados por la investigación en el ámbito de la educación inclusiva. Revista Latinoamericana De Educación Inclusiva, 8(2), 25-48.

Escudero, J. M. \& Martínez, B. (2011). Educación inclusiva y cambio escolar. Revista Iberoamericana De Educación, 55(1), 85-105.

Etxabe, E., López-Vélez, A.L. \& Soria, L. (2018). Escuela Inclusiva: únete al reto. Bilbao: FEVAS.

Fiuza, M. J. \& Sierra, S. (2017). Creando y adaptando materiales para compartir: una experiencia con futuros maestros. Educatio Siglo XXI: Revista De La Facultad De Educación, 35(3), 153-174.

García Rubio, J. (2017). Evolución legislativa de la educación inclusiva en España. Revista De Educación Inclusiva, 10(1), 251-264.

Garrido, A. (2012). Justicia social en la dialéctica inclusión/exclusión social. Revista Integra Educativa, 5(2), 1997-4043.

Giroux, H.A. (2009). El reto y promesa de la pedagogía crítica en la nueva era de la información: una entrevista con Henry Giroux. Education in the Knowledge Society (EKS), 10(3), 243-255.
Giroux, H.A. (2015). Pedagogías disruptivas y el desafío de la justicia social bajo regímenes neoliberales. Revista Internacional De Educación Para La Justicia Social (RIEJS), 4(2), 13-27.

Giroux, H.A. (2016). La educación superior y las políticas de ruptura. Entramados: Educación $Y$ Sociedad, 3, 15-26.

Karsz, S. (2004). La exclusión: concepto falso, problema verdadero, en S., Karsz (Coord.). La exclusión: bordeando sus fronteras. Definiciones y matices (pp. 133-214). Barcelona: Gedisa.

Lodi, M. (1977). El país errado. Barcelona: Editorial Laia.

McLaren, P. \& Kincheloe, J. (2008). Pedagogía crítica: de qué hablamos, dónde estamos. Barcelona: Graó.

McLaren, P. (2005). La vida en las escuelas: una introducción a la pedagogía crítica en los fundamentos de la educación. Buenos Aires: Siglo XXI Editores, S.A.

Muntaner, J.J., Rossello, M.R. \& De la Iglesia, B. (2016). Buenas Prácticas en Educación Inclusiva. Educatio Siglo XXI, 34(1), 31-49.

Nussbaum, M.C. (2018). Las fronteras de la justicia: consideraciones sobre la exclusión. Barcelona: Paidós.

Parrilla, A. (2002). Acerca del origen y sentido de la Educación Inclusiva. Revista de Educación, 327, 11-29.

Parrilla, A. (2013). Presentación. Equidad e Innovación en la investigación educativa: reflexiones y aportaciones desde la red de investigación CIES. Revista de investigación en educación, 3(11), 7-13.

Pérez, M.E. (2017). Aulas inclusivas: experiencias prácticas. Tarragona: Altaria.

Peterson, B. (2005). La Escuela Fratney: un viaje hacia la democracia. En M.W. Apple \& J.A. Beane (Comps.), Escuelas democráticas (pp. 95-130). Madrid: Morata.

Rodríguez, H., Jiménez, M. \& Sánchez, S. (2018). Construcción del discurso en torno a la educación inclusiva. Revista Electrónica Interuniversitaria De Formación Del Profesorado, 21(1), 185-217. 
Rubia, F.A. (2017). ¿Puede ser inclusiva la escuela en un sistema segregador? Forum Aragón: Revista Digital De FEAE-Aragón Sobre Organización y Gestión Educativa, 22, 85-91.

Sánchez, A. \& Jiménez, M. (2013). Exclusión social: fundamentos teóricos y de la intervención. Trabajo Social Global, 3(4), 133-156.

Sen, A. (2000). Social Exclusion: Concept, application, and scrutiny. Philippines: Asian Development Bank.

Slee, R. (2012). La escuela extraordinaria: exclusión, escolarización y educación inclusiva. Madrid: Morata.

TONUCCI, F. (2005). Con ojos de niño. Buenos Aires: Losada.

UNESCO. (2012). Lucha contra la exclusión en la educación: guía de evaluación de los sistemas educativos rumbo a sociedades más inclusivas $y$ justas. Geneve: International Bureau of Education/Unesco.
UNESCO. (2017a). Resumen del Informe de seguimiento de la educación en el mundo 2017/18. Reducir cuentas en el ámbito de la educación. Cumplir nuestros compromisos. París: Organización de las Naciones Unidas para la Educación, la Ciencia y la Cultura.

UNESCO (2017b). Guia para asegurar la inclusión y la equidad en la educación. París: Organización de las Naciones Unidas para la Educación, la Ciencia y la Cultura.

UNESCO (2018). Unesco 2017. Paris: United Nations Educational, Scientific and Cultural Organization.

UNICEF (2014). Teachers, Inclusive, Child-Centred Teaching and Pedagogy. New York: Unicef.

UNICEF (2017). Inclusive Education. Understanding article 24 of the Convention on the Rights of persons with disabilities. Geneva: Unicef.

\section{Agradecimientos}

Este trabajo se enmarca en el Grupo de investigación del Gobierno Vasco «KideOn», con referencia IT1342-19 (categoría A); Redes de Innovación para la Inclusión Educativa y Social: Infancia Vulnerable, Servicios Socioeducativos y Familias (EDU2015-68617-C4-2-R) (MINECO/FEDER, UE). 